\title{
Circulations et échanges de plantes et de savoirs phyto-médicinaux sur la frontière franco- brésilienne
}

Marc-Alexandre Tareau, Lucie Dejouhanet, Marianne Palisse et Guillaume Odonne

\section{OpenEdition}

\section{Journals}

Édition électronique

URL : http://journals.openedition.org/rfst/382

DOI : $10.4000 /$ rfst.382

ISSN : 2492-3672

Éditeur

Espaces et SOciétés (UMR 6590)

Référence électronique

Marc-Alexandre Tareau, Lucie Dejouhanet, Marianne Palisse et Guillaume Odonne, « Circulations et échanges de plantes et de savoirs phyto-médicinaux sur la frontière franco-brésilienne », Revue francophone sur la santé et les territoires [En ligne], Les circulations en santé : des produits, des savoirs, des personnes en mouvement, mis en ligne le 16 décembre 2019, consulté le 06 avril 2021. URL http://journals.openedition.org/rfst/382 ; DOI : https://doi.org/10.4000/rfst.382

Ce document a été généré automatiquement le 6 avril 2021.

\section{cc) (1) (ㅇ)}

La Revue francophone sur la santé et les territoires est mise à disposition selon les termes de la Licence Creative Commons Attribution - Pas d'Utilisation Commerciale - Partage dans les Mêmes Conditions 4.0 International. 


\title{
Circulations et échanges de plantes et de savoirs phyto-médicinaux sur la frontière franco-brésilienne
}

\author{
Marc-Alexandre Tareau, Lucie Dejouhanet, Marianne Palisse et Guillaume \\ Odonne
}

Ce travail a été financé par l'appel à projets de l'Observatoire Hommes-Milieux "Oyapock", et a également bénéficié $d u$ financement des projets "investissements d'avenir" de l'Agence Nationale de la Recherche (Labex DRIIHM/IRDHEI et Labex CEBA: ANR-10-LABX-25-01).

Nous souhaitons remercier chaleureusement la population de Saint-Georges-de-l'Oyapock qui s'est prêtée au jeu des enquêtes.

\section{Introduction et méthodologie}

\section{Des « pharmacopées sans frontières »}

1 Depuis les travaux de Charles Leslie (Leslie, 1989, 1980 ; Leslie et al., 1992) et de Jean Benoist (Benoist, 1996) sur le pluralisme médical et la construction des savoirs médicaux, les systèmes thérapeutiques dits «traditionnels " ne sont plus considérés comme figés, mais bien comme des médecines dynamiques, en perpétuelle évolution et ouvertes à des influences culturelles diverses. Ces systèmes de soins empruntent les uns aux autres concepts et matières médicales et se modifient donc au contact d'autres thérapies, de la biomédecine, des sciences, des technologies, en lien avec les choix politiques et économiques de valorisation les concernant (Craig, 2013; Ladio \& Albuquerque, 2014 ; Leonti, 2011 ; Pordié \& Simon, 2013). Les savoirs thérapeutiques se nourrissent ainsi des circulations multiples et des interactions entre les groupes culturels, autant en milieu urbain (Ceuterick et al., 2008; Ladio \& Albuquerque, 2014 ; Tareau et al., 2017) qu'en milieu rural, voire isolé (Alexiades, 2009; Leonti, 2011; Odonne et al., 2011). En effet, à l'image d'autres types de circulations transnationales qui accompagnent souvent les populations dispersées sur plusieurs territoires (Audebert, 2017 ; Bruneau, 2010 ; Mung, 1992), les plantes médicinales ont tendance à 
suivre les groupes diasporiques (Ososki et al., 2007 ; Pieroni et al., 2008). Cela donne lieu à des mutations des pratiques thérapeutiques qui oscillent entre recomposition et conservatisme (Vandebroek \& Balick, 2012 ; Volpato et al., 2008) avec l'incorporation, la disparition ou la conservation de certaines espèces dans la pharmacopée des diasporas, en fonction de leurs stratégies d'adaptation et d'intégration, des fonctionnements cognitifs ou de motifs culturels particuliers (Ceuterick et al., 2011, 2008).

Dans ce contexte, les espaces transfrontaliers constituent des lieux d'échanges et d'interactions « interstitiels » (Ruffray, 2000) dans lesquels les pratiques culturelles se confrontent, se mélangent et peuvent aussi prendre des formes singulières (de Oliveira, 2009). Les circulations et mobilités thérapeutiques sur les frontières composent ainsi un "champ de recherche en construction" (Sakoyan, 2012) qui s'est surtout focalisé jusqu'à présent sur les migrations des malades pour un recours aux soins biomédicaux (Bochaton et Lefebvre, 2010, 2008; Pordié, 2013) ou sur la patrimonialisation des pratiques traditionnelles de soins comme stratégie de reconnaissance d'identités culturelles transfrontalières (Candelise, 2013; Carreño Calderón, 2013). Les flux transnationaux de plantes commencent aujourd'hui à susciter également l'intérêt, notamment avec la diffusion des médecines alternatives dans les pays occidentaux (Dejouhanet, 2009; Hoyez, 2011), la demande des membres de diasporas en remèdes à base de plantes de leur région d'origine (Balick et al., 2000 ; Bochaton, 2018 ; Ceuterick et al., 2008 ; Kujawska \& Hilgert, 2014 ; Pieroni et al., 2012 ; Reiff et al., 2003 ; van Andel \& Westers, 2010), et le développement des industries pharmaceutiques dites traditionnelles dans les pays du Sud (Saxer, 2009; Dejouhanet, 2014 ; Mercan, 2012). Encore trop peu d'études analysent dans les espaces transfrontaliers les dynamiques de ces flux, leur rôle dans la transformation des pratiques thérapeutiques et plus globalement, la manière dont les savoirs et les plantes circulent et s'implantent à ces interfaces.

\section{La frontière franco-brésilienne : une limite géopolitique au cœur d'un ensemble culturel}

En Guyane, les fleuves frontières (Maroni à l'ouest, Oyapock à l'est) sont des lieux de contacts et d'échanges plus que de franche séparation. Les fleuves, en contexte amazonien, constituent des axes de circulation qui structurent les échanges aussi bien entre le littoral et l'intérieur des terres qu'au sein de l'espace continental, contribuant à une construction territoriale particulière. Comme ailleurs, les fleuves sont «des corridors de transports, des sites pour la création de villes, des limites spatiales ou des zones d'échanges » (Bruckmann, 2017), parfois centraux pour les territoires nationaux, parfois marginalisés, surtout lorsqu'ils correspondent aux limites géopolitiques. Les utiliser comme frontière est alors toujours un peu « paradoxal » (Théry, 2015).

4 Sur l'Oyapock, fleuve-frontière entre le Brésil et la Guyane française, deux pays se font face, avec leurs spécificités, leurs valeurs et leurs codes culturels. Pourtant, comme l'écrit René Nouailhat (2010), il s'agit d'une "frontière impossible ». Ceci, à la fois pour des raisons historiques (le tracé de la frontière fit l'objet d'un long conflit diplomatique entre la France et le Brésil résolu seulement en 1900 au profit du Brésil par un arbitrage suisse) et géographiques (un vaste fleuve amazonien n'est pas une frontière imperméable). Il en découle une proximité culturelle - notamment linguistique ${ }^{1}$ - et une homogénéité des modes de vie entre les populations de l'Amapa et de la Guyane 
française. Les va-et-vient permanents entre les deux rives sont l'illustration la plus concrète de l'extrême porosité de cette frontière ${ }^{2}$ qui agit de fait comme une véritable « interface dynamique entre deux mondes » (Blancodini \& Tabarly, 2010), d'autant que ces «deux mondes" ne sont séparés politiquement que depuis un siècle (Grenand, 2012). Les circulations entre les deux rives ont précédé la présence des colons dans la région à partir du 16e siècle et n'ont cessé de s'intensifier par la suite (Collomb \& van den Bel, 2014; Dupuy, 2012). Encore aujourd'hui, les deux rives fonctionnent en synergie : les habitants de Saint-Georges-de-l'Oyapock traversent le fleuve pour acheter des biens de consommation à Oiapoque, des Français habitent sur la berge opposée pour bénéficier de loyers modestes, les Brésiliens se rendent au dispensaire de SaintGeorges pour des consultations médicales, etc. (Boudoux d'Hautefeuille, 2008). Autant de mobilités quotidiennes ou fréquentes qui contribuent au dynamisme circulatoire de la zone.

5 Ce co-fonctionnement des deux rives frontalières du bas-Oyapock, appuyé par les nombreuses analogies culturelles et également naturelles ${ }^{3}$, est d'autant plus marqué que cette région fut pendant longtemps enclavée. Les deux rives se ressemblent ainsi de par leur marginalité vis-à-vis du pouvoir central de leurs États respectifs et de par leur désenclavement récent ${ }^{4}$ (fig. 1) : Saint-Georges-de-l'Oyapock est à trois heures de route de Cayenne et à plus de huit heures de vol de Paris. Oiapoque est au mieux à huit heures de route de Macapa, la capitale de l'État de l'Amapa, et à $1600 \mathrm{~km}$ de Brasilia. Ce relatif isolement a évidemment influencé leur fonctionnement commun et l'histoire collective de ce qui était autrefois un territoire singulier.

6 Cependant, en dépit d'une cohérence historique, le fossé socio-économique entre les deux rives se creuse aujourd'hui : la rive gauche est européenne (la Guyane est un département d'outre-mer depuis 1946) et la rive droite est Amapaense, l'Amapa étant l'un des États les plus pauvres et les plus enclavés du Brésil (il n'est relié à aucun autre État par la route). Le niveau de vie relativement élevé et l'assistance sociale garantie par l'État français induisent un important différentiel socio-économique frontalier, source de migrations brésiliennes importantes vers une Guyane française perçue comme très attractive (Police, 2010). Jusqu'à récemment les habitants se rendaient d'un côté comme de l'autre sans contrainte administrative (Grenand, 2012), les titres de séjour n'étant contrôlés que sur les barrages routiers installés du côté français sur la route de Cayenne et du côté brésilien à la sortie d'Oiapoque, en direction de Macapa, ce qui créait de facto un espace commun (Nicolas, 2016). Mais le bras de fer croissant entre l'Union Européenne et le Mercosur ainsi que les législations fort différentes de ces deux grands ensembles politiques et économiques font apparaitre une ligne de démarcation de plus en plus rigide, avec des contrôles nombreux et contraignants d'un côté comme de l'autre. L'ouverture en 2017 du pont transnational sur l'Oyapock dont le passage oblige à un double-contrôle systématique par les autorités douanières et policières des deux pays, contribue également au processus en cours de refrontièrisation ( rebordering ) (Letniowska-Swiat, 2012; Moullé, 2017). Quoiqu'il en soit, de nombreux déplacements pendulaires (Kaufmann, 2005) se font entre les deux rives et contribuent par essence à la fabrique de cet espace culturel (Crété, 2015 ; Filippi, 2016; Thebaux, 2015 ; Tritsch et al., 2012).

7 La région est donc depuis longtemps le lieu de circulations et d'échanges culturels soutenus, parmi lesquelles les échanges liés aux pratiques de santé constituent un vaste champ de recherche. Notre étude a pour but d'identifier et de caractériser les 
circulations ethnobotaniques ${ }^{5}$ dans le bas-Oyapock et en particulier à Saint-Georgesde-l'Oyapock, ces circulations étant autant de preuves de la dimension culturelle profonde des échanges sur cette frontière. En effet, si ce travail apporte un éclairage original sur des formes de circulations informelles et relativement méconnues, il permet aussi, par son entrée ethnobotanique, de mieux comprendre les constructions culturelles qui s'opèrent dans ces types d'espaces.

Figure 1 : Contexte géographique régional de la frontière franco-brésilienne, 2018

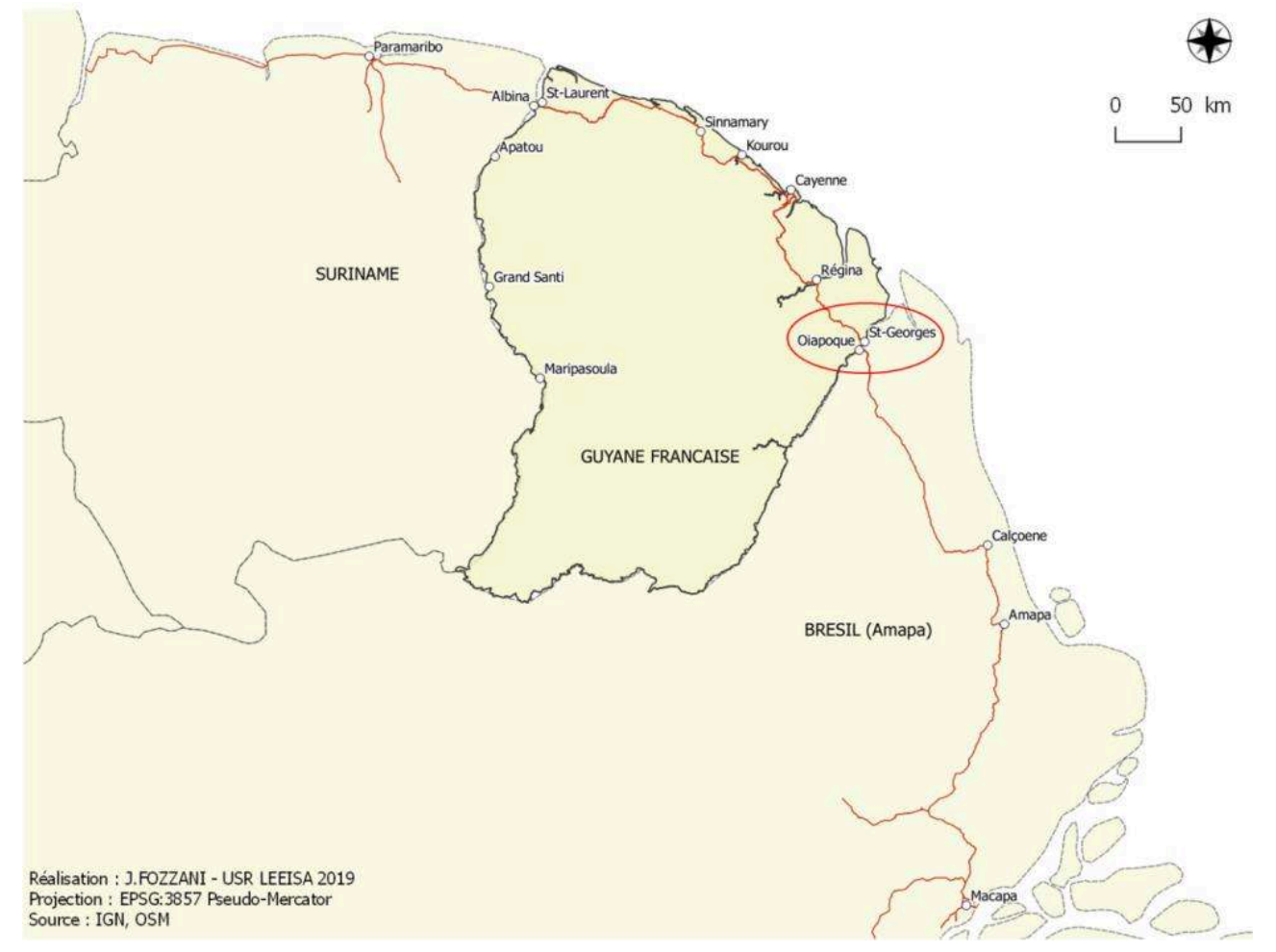

\section{Mesurer les échanges de plantes et de savoirs sur l'Oyapock : méthodologie}

8 Dans le bassin du bas-Oyapock, plusieurs groupes humains cohabitent et se déplacent régulièrement d'une rive à l'autre du fleuve-frontière. Ce travail repose uniquement sur des enquêtes réalisées à Saint-Georges-de-l'Oyapock ${ }^{6}$ durant plusieurs missions de terrain entre octobre 2016 et avril 2017. Des entretiens semi-directifs y ont été menés avec vingt-trois habitants, tous utilisateurs plus ou moins réguliers de plantes médicinales.

9 Ces enquêtes ont été effectuées dans une période de vide législatif, entre la promulgation de la loi pour la reconquête de la biodiversité ${ }^{7}$ et l'entrée en vigueur de son décret d'application, le 1er juillet 2017. L'instance chargée par le ministère de la Transition écologique et solidaire de délivrer les autorisations pour les projets concernés par l'accès aux ressources génétiques et le partage juste et équitable des avantages (APA) n'avait alors pas été mise en place ${ }^{8}$. Le comité APA de la collectivité territoriale de Guyane (qui s'était attribué la compétence avant l'émission d'une loi nationale) a arrêté de siéger à la parution de la loi biodiversité. En l'absence de modalités d'application, nous avons procédé en accord avec les principes du code 
d'éthique de la Société internationale d'Ethnobiologie ${ }^{9}$, de la manière suivante: les autorités coutumières des Amérindiens palikur ont été rencontrées en amont du travail de doctorat dans lequel s'inscrit cette étude en la personne de Roger Labonté et d'Emmanuel Guillaumet et leur accord filmé a été préalablement recueilli. Il faut enfin souligner que le présent article ne concerne pas le contenu des "recettes" des remèdes, mais bien la circulation des plantes et des savoirs. Les habitants interviewés appartenaient aux cinq principaux groupes culturels présents dans la région du basOyapock (six Amérindiens palikur, six Créoles guyanais, six Brésiliens non amérindiens, trois Amérindiens galibi-marwono et deux Amérindiens karipuna). Ils ont été rencontrés au hasard, lors de nos incursions dans les différents quartiers de la commune (voir carte) qui se caractérisent - à l'exception des nouveaux logements de type Habitation à Loyer Modéré et, dans une certaine mesure, du vieux bourg - par leur composition souvent mono-ethnique (Pérez and Archambeau, 2012) (fig. 2). Les Amérindiens palikur, de langue arawak, ont ainsi été rencontrés dans les quartiers Espérance, Martin et Baptiste, à Gabin et Petite-Savane. Leur population dans la région est estimée à 3000 personnes (Laval, 2016) réparties dans différents villages, tant du côté français que brésilien (villages de la région du fleuve Urucauà). Les GalibiMarwono et Karipuna, issus de groupes amérindiens comptant entre 2000 et 3000 membres à l'échelle régionale ( $i b i d$.) et principalement issus de la fusion d'autres groupes aujourd'hui éteints, ont été interviewés respectivement à Onozo et Petitesavane, et dans le Village Bambou et le quartier Maripa. Même si des familles habitent en Guyane, ces groupes sont principalement installés au Brésil, dans la région du fleuve Uaçà pour les Galibi-marwono et du fleuve Curipi pour les Karipuna (fig. 2). Les Brésiliens non-amérindiens habitant la région du bas-Oyapock, sont issus d'une migration plus ou moins récente de populations urbaines pauvres en provenance soit des villes du sud de l'Amapa soit des États voisins du Parà, du Maranhão voire du Céara (Boudoux d'Hautefeuille, 2012). Ils sont principalement implantés dans la ville frontière d'Oiapoque (15 000 habitants), mais sont également depuis quelques années très enclins à migrer vers la Guyane ${ }^{10}$ pour y trouver du travail et tenter d'améliorer leurs conditions d'existence. Nous en avons rencontré dans le bourg de Saint-Georges, ainsi que dans les quartiers Petite-Savane, Gabin et Onozo. Enfin, les Créoles guyanais, descendants d'esclaves qui se sont dispersés sur les anciennes terres des colons suite à l'abolition de 1848, habitent principalement dans le centre-bourg où nous les avons rencontrés, ainsi que dans le quartier Blondin pour les Créoles d'origine saamaka (businenge).

Plusieurs thématiques ont été abordées avec les personnes rencontrées :

- Afin de pouvoir dresser un inventaire des plantes médicinales en usage à Saint-Georges-del'Oyapock, nous avons demandé à chaque personne de citer l'ensemble des espèces qu'elle connaissait et utilisait pour se soigner. Parallèlement, nous avons collecté des herbiers ${ }^{11}$ pour déterminer précisément les espèces citées, car, dans ce contexte fortement multilingue, les très nombreux noms vernaculaires introduisent beaucoup d'imprécisions. Les citations des usages ( Use Reports, URs) de chacune des plantes mentionnées ont été répertoriées et permettent de mesurer, conformément à l'explication donnée par 0 . Phillips et A.H. Gentry (1993), la fréquence de citations d'une même espèce, en général ou dans un cadre thérapeutique particulier.

- Nous avons également interrogé les personnes sur la provenance du ou des savoir(s) concernant chacune des plantes citées (lien relationnel, sexe et communauté d'appartenance de chacun des transmetteurs) dans l'objectif de comprendre et de quantifier 
les modalités de transmission et de mesurer la porosité ou l'imperméabilité aux savoirs exogènes des communautés transfrontalières. Ces interrogations spécifiques ont également permis de mesurer l'importance des circulations de plantes et de produits à base de plantes sur l'espace transfrontalier ainsi que les éventuels processus d'hybridation ethnobotanique (Ladio \& Albuquerque, 2014) en contexte multiculturel.

- Enfin, nous avons voulu mieux définir les flux et mobilités liés aux plantes et leur incidence dans les phytothérapies locales et nous avons demandé des précisions sur le lieu d'approvisionnement des plantes utilisées. Une visite dans la ville frontalière d'Oiapoque nous a permis d'observer un certain nombre de plantes médicinales et de produits phytomédicinaux commercialisés dans les herboristeries et sur le marché et d'apporter quelques éléments de compréhension complémentaires des modalités d'acheminement de ces produits.

11 À la lumière des résultats de cette enquête, nous proposons une description des pratiques et des savoirs de phytothérapie contemporains dans la région de SaintGeorges-de-l'Oyapock (II) ainsi que des circulations humaines et commerciales qui les nourrissent et participent à leur dynamisme (III).

Figure 2 : Carte des quartiers de Saint-Georges-de-l'Oyapock dans lesquels ont eu lieu les enquêtes et des communautés y résidant.

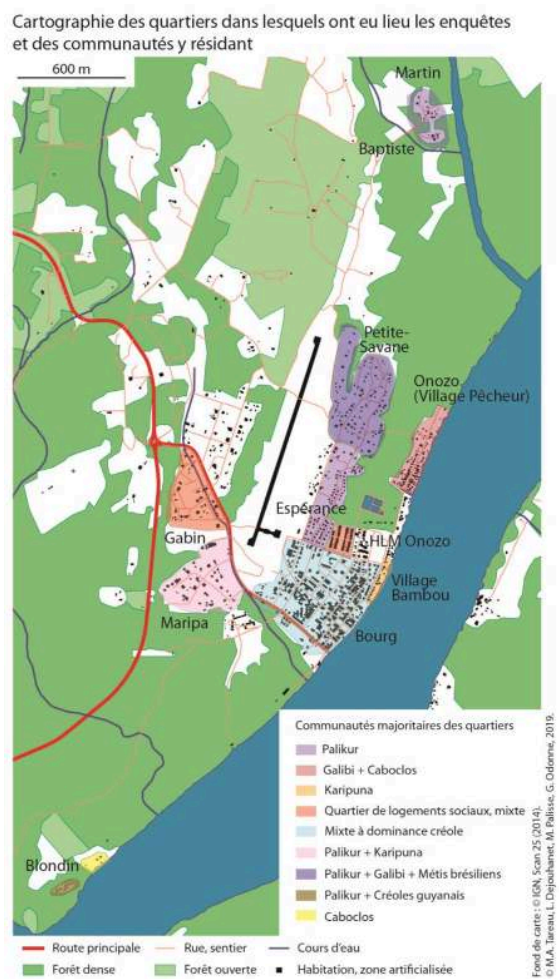




\section{Le paysage phyto-thérapeutique de la petite ville frontalière de Saint-Georges-de-I'Oyapock}

\section{Les plantes de Saint-Georges-de-l'Oyapock, un assemblage botanique hétéroclite}

Le paysage phyto-thérapeutique guyanais est caractérisé dans son ensemble, par une importante diversité de pratiques, reflétant elles-mêmes l'importante diversité socioculturelle de ce territoire (Tareau et al., 2018, 2017). De nombreuses espèces natives et exotiques, en particulier d'Asie et d'autres régions d'Amérique (tab.1), sont utilisées avec des fonctions et selon des modes de préparation qui peuvent différer en fonction de l'appartenance culturelle des habitants. Des analogies ou des spécificités plus ou moins marquées peuvent ainsi être observées dans les usages entre les différentes communautés présentes sur le territoire. L'inventaire effectué au début des années 1980 par P. Grenand et al. (1986, rééd. 2004) auprès des communautés créole, palikur et teko de Guyane recensait 620 espèces potentiellement utilisées pour leurs usages thérapeutiques.

13 À Saint-Georges-de-l'Oyapock, ce sont au total 139 espèces qui ont été citées lors de l'enquête, totalisant 410 citations d'usages (URs). Parmi elles, 52 espèces totalisant 294 citations, et appartenant à 26 familles botaniques, ont une récurrence d'au moins trois citations, pouvant ainsi être considérées comme les espèces les plus significatives de cette pharmacopée.

Tableau 1 : Liste des 52 principales espèces médicinales citées à Saint-Georges-de-l'Oyapock.

\begin{tabular}{|l|l|l|l|l|}
\hline Espèce & Famille & Citations & $\begin{array}{l}\text { Statut } \\
\text { agronomique }\end{array}$ & Origine \\
\hline Quassia amara L. & Simaroubaceae & 16 & C & Amérique \\
\hline Carapa guianensis Aubl.* & Meliaceae & 14 & S & Amérique \\
\hline Astrocaryum vulgare Mart.* & Arecaceae & 13 & S & Amérique \\
\hline Copaifera sp.* & Fabaceae & 12 & S & Amérique \\
\hline Eryngium foetidum L. & Apiaceae & 12 & C & Amérique \\
\hline $\begin{array}{l}\text { Citrus x aurantiifolia (Christm.) } \\
\text { Swingle }\end{array}$ & Rutaceae & 11 & C & Asie \\
\hline Cymbopogon citratus (DC.) Stapf & Poaceae & 11 & C & Asie \\
\hline $\begin{array}{l}\text { Ayapana triplinervis (Vahl) R.M.King } \\
\text { \& H.Rob. }\end{array}$ & Asteraceae & 10 & C & Amérique \\
\hline Leonotis nepetifolia (L.) R.Br. & Lamiaceae & 9 & C & Amérique \\
\hline $\begin{array}{l}\text { Gymnanthemum } \\
\text { (Delile) Sch.Bip. ex Walp. }\end{array}$ & Asteraceae & 8 & C & Afrique \\
\hline Cocos nucifera L.* & Arecaceae & 8 & C & Asie \\
\hline Bryophyllum pinnatum (Lam.) Oken & Crassulaceae & 8 & C & Afrique \\
\hline Petiveria alliacea L. & Phytolaccaceae & 8 & C & Amérique \\
\hline
\end{tabular}




\begin{tabular}{|c|c|c|c|c|}
\hline Siparuna guianensis Aubl. & Siparunaceae & 8 & $\mathrm{~S}$ & Amérique \\
\hline Aloe vera (L.) Burm.f. & Xanthorrhoeaceae & 7 & C & Amérique \\
\hline Chenopodium ambrosioides L. & Amaranthaceae & 6 & c & Amérique \\
\hline Morinda citrifolia $\mathrm{L}$. & Rubiaceae & 6 & C & Océanie \\
\hline Anacardium occidentale L. & Anacardiaceae & 5 & C & Amérique \\
\hline Allium sativum $\mathrm{L}$. & Amaryllidaceae & 5 & C & Europe \\
\hline $\begin{array}{l}\text { Cinnamomum zeylanicum Garcin ex } \\
\text { Blume }\end{array}$ & Lauraceae & 5 & C & Asie \\
\hline Justicia pectoralis Jacq. & Acanthaceae & 5 & C & Amérique \\
\hline Mansoa alliacea (Lam.) A.H.Gentry & Bignoniaceae & 5 & $\mathrm{c} / \mathrm{s}$ & Amérique \\
\hline Momordica charantia L. & Cucurbitaceae & 5 & $S$ & Asie \\
\hline Ocimum spp. & Lamiaceae & 5 & C & $\begin{array}{l}\text { Asie/Amériq } \\
\text { ue }\end{array}$ \\
\hline Picrolemma sprucei Hook. F. & Simaroubaceae & 5 & $S$ & Amérique \\
\hline Costus spiralis (Jacq.) Roscoe & Costaceae & 4 & $\mathrm{~S}$ & Amérique \\
\hline Hibiscus rosa-sinensis L. & Malvaceae & 4 & C & Asie \\
\hline Jatropha curcas L. & Euphorbiaceae & 4 & C & Amérique \\
\hline $\begin{array}{l}\text { Lippia alba (Mill.) N.E.Br. ex Britton } \\
\text { \& P.Wilson }\end{array}$ & Verbenaceae & 4 & C & Amérique \\
\hline Lantana camara L. & Verbenaceae & 4 & $\mathrm{~s}$ & Amérique \\
\hline Manihot esculenta Crantz & Euphorbiaceae & 4 & C & Amérique \\
\hline $\begin{array}{l}\text { Tinospora crispa (L.) Hook. f. \& } \\
\text { Thomson }\end{array}$ & Menispermaceae & 4 & C & Asie \\
\hline $\begin{array}{l}\text { Virola surinamensis (Rol. ex Rottb.) } \\
\text { Warb. }\end{array}$ & Myristicaceae & 4 & $\mathrm{~s}$ & Amérique \\
\hline Dalbergia monetaria L. $\mathrm{f}$. & Fabaceae & 4 & $S$ & Amérique \\
\hline Averrhoa bilimbi L. & Oxalidaceae & 3 & C & Asie \\
\hline Alternanthera brasiliana (L.) Kuntze & Amaranthaceae & 3 & C & Amérique \\
\hline Annona muricata $\mathrm{L}$. & Annonaceae & 3 & C & Amérique \\
\hline Banara guianensis Aubl. & Salicaceae & 3 & $S$ & Amérique \\
\hline Vetiveria zizanioides (L.) Nash & Poaceae & 3 & C & Amérique \\
\hline Gossypium barbadense L. & Malvaceae & 3 & C & Amérique \\
\hline Geissospermum laeve (Vell.) Miers & Apocynaceae & 3 & $S$ & Amérique \\
\hline Myristica fragrans Houtt. & Myristicaceae & 3 & C & Asie \\
\hline Mangifera indica $\mathrm{L}$. & Anacardiaceae & 3 & C & Asie \\
\hline Mentha spp. & Lamiaceae & 3 & C & Europe \\
\hline Nicotiana tabacum L. & Solanaceae & 3 & C & Amérique \\
\hline
\end{tabular}




\begin{tabular}{|l|l|l|l|l|}
\hline Opuntia cochenillifera (L.) Mill. & Cactaceae & 3 & C & Amérique \\
\hline Fraxinus ornus L.* & Oleaceae & 3 & S & Europe \\
\hline Psidium guajava L. & Myrtaceae & 3 & C & Amérique \\
\hline Ricinus communis L. & Euphorbiaceae & 3 & C & Afrique \\
\hline Senna alexandrina Mill. & Fabaceae & 3 & C & Afrique \\
\hline Zingiber officinale Roscoe & Zingiberaceae & 3 & C & Asie \\
\hline Indéterminée. & NP & 3 & S & Amérique \\
\hline
\end{tabular}

Légende : * = commercialisé sous forme d'un produit transformé ; C = cultivée ; S = sauvage.

14 Un cinquième de ces 52 plantes sont des espèces natives du bassin amazonien ou du plateau des Guyanes (tab. 1), totalisant $26 \%$ du total des citations. La grande majorité des plantes utilisées $(79 \%)$ sont par conséquent des espèces exotiques (dont l'usage est souvent d'ailleurs pantropical). Enfin, seulement $8 \%$ des espèces ne sont utilisées que par une seule et même communauté, la majorité des plantes étant employée de façon transverse par plusieurs communautés. Sur ces plantes partagées, $52 \%$ le sont entre deux ou trois communautés et $36 \%$ sont utilisées par quatre ou cinq des communautés consultées, dressant déjà un premier tableau d'un patrimoine phyto-thérapeutique commun.

Photographie 1 : Jardin de plantes médicinales le long d'une maison. Une grande partie des savoirs sur les plantes et leurs usages est transmise par les femmes. Village Bambou, 2017.

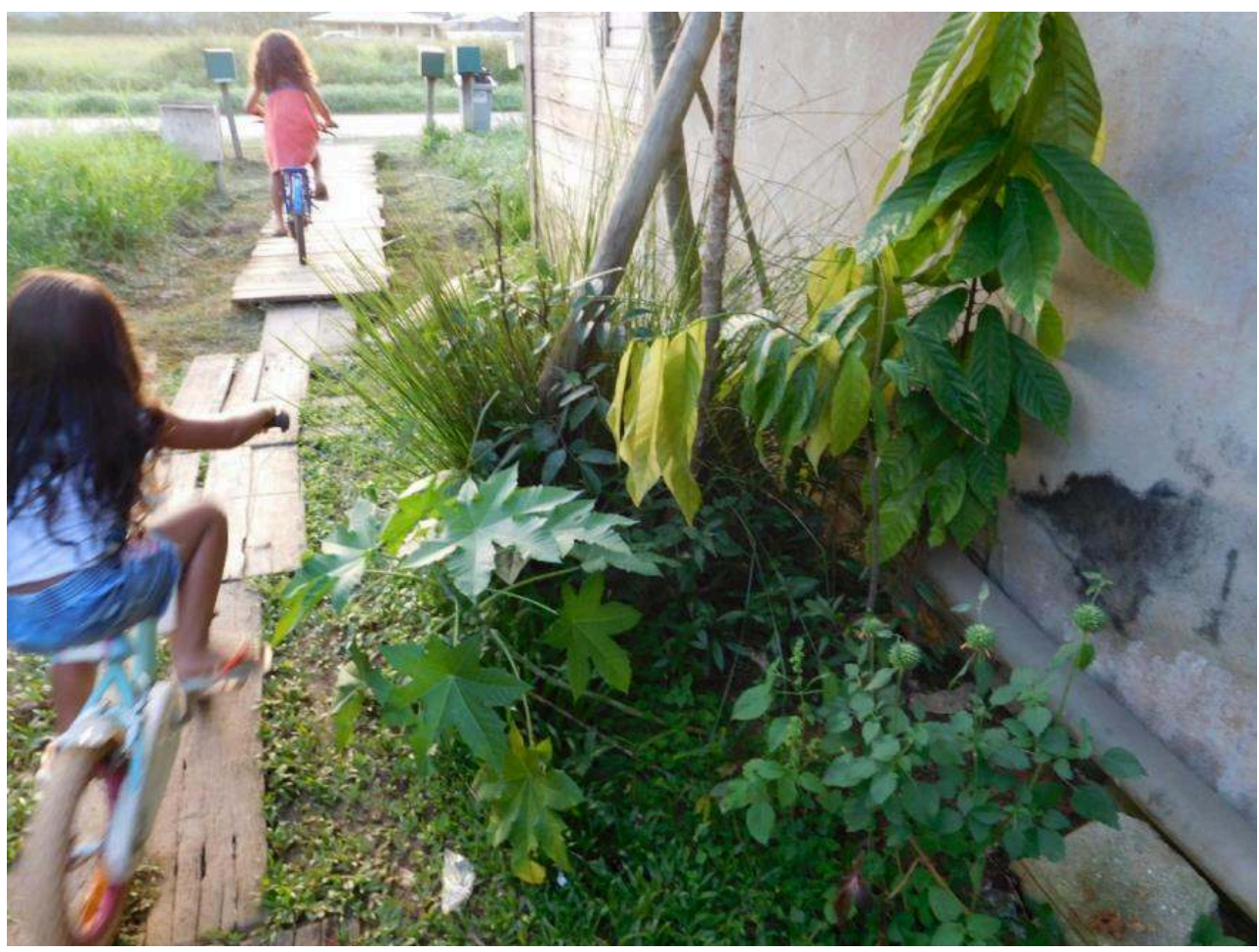




\section{L'origine de la connaissance : les savoirs et leur circulation entre les communautés}

15 Malgré cette utilisation transverse d'un grand nombre de plantes, la transmission des savoirs liés aux plantes médicinales dans la région de Saint-Georges-de-l'Oyapock demeure essentiellement intra-communautaire et intergénérationnelle (transmission verticale) avec une place prépondérante des femmes dans ce processus ${ }^{12}$ (et ceci, tant dans la transmission faite aux femmes qu'aux hommes) (photo 1). En effet, sur un total de 410 citations de plantes médicinales, 349 correspondent à une transmission verticale et 154 à une transmission de la mère, la grand-mère ou une tante ${ }^{13} .58$ citations $(14,1 \%)$ correspondent à une transmission horizontale qui peut être interfamiliale ou intercommunautaire, et enfin 3 seulement des citations de plantes ont pour origine les médias.

Bien qu'il soit relativement faible, le degré de perméabilité aux échanges interculturels, de chacune des communautés est tout de même intéressant à explorer. En croisant l'origine culturelle des informateurs et des personnes qui leur ont transmis les connaissances citées, nous pouvons dresser des profils communautaires en fonction du niveau de perméabilité aux savoirs allochtones ou de la capacité de diffusion de ses propres savoirs vers les autres groupes culturels (fig. 3). Les personnes cherchent certes d'abord dans leur propre communauté l'information (à plus de $70 \%$ ), mais il semble, avec toutes les précautions qu'implique la taille de notre échantillon, que les groupes sans doute les moins socialement isolés - car étant les plus anciennement implantés dans la zone - (Palikur, Créoles, Brésiliens) sont les plus enclins à échanger en dehors de leur propre communauté, en agissant comme les principaux groupes "ressource " de l'information obtenue. Les Galibi-marwono et Karipuna semblent alors relativement assimilateurs de savoirs exogènes, mais peu enclins à partager leurs propres connaissances avec les autres communautés. 
Figure 3 : Échanges de savoirs phyto-médicinaux à Saint-Georges de l'Oyapock : perméabilité aux savoirs allochtones et capacité de diffusion chez les communautés de Saint-Georges. Réalisé sur la base d'un schéma proposé par Sébastien Oliveau.

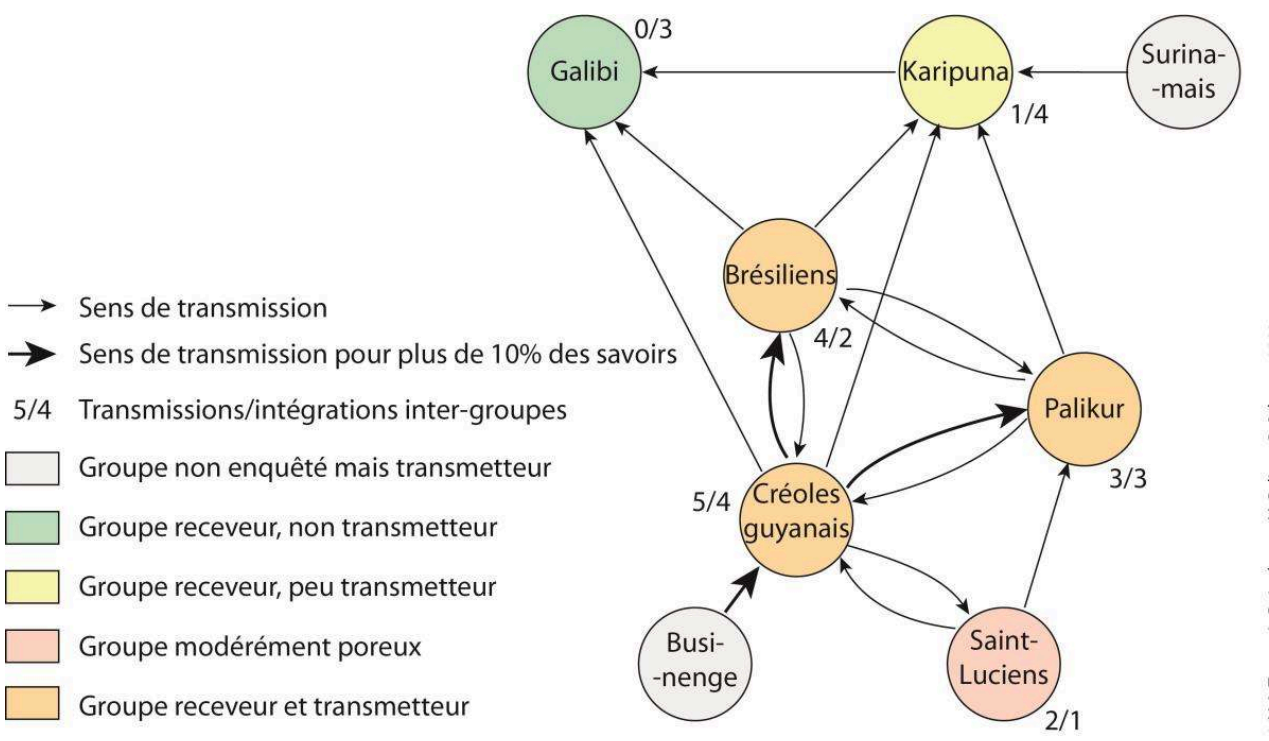

Explication : Aucune information n'a été relevée comme venant des Galibi-marwono parmi les savoirs des autres communautés, par contre ce groupe amérindien a intégré dans sa pharmacopée des connaissances venant des Karipuna, des Brésiliens et des Créoles guyanais : il est donc associé à une fraction 0/3. Les Brésiliens interrogés ont reçu plus de $10 \%$ de leurs savoirs recensés des Créoles guyanais, tout en leur transmettant aussi des connaissances ; la fraction 4/2 indique qu'ils ont partagé leurs connaissances avec quatre communautés et reçus des informations de deux autres groupes. Ils sont donc à la fois receveur et transmetteur, au contraire des Galibi-marwono qui ne sont que receveurs.

Ces échanges de savoirs entre communautés ont vraisemblablement contribué à l'utilisation généralisée d'un grand nombre de plantes. Si le portrait que nous en traçons correspond aux circulations relativement récentes entre les communautés, il est évident que ces échanges, qui ont contribué à enrichir les pharmacopées locales, sont à intégrer dans un temps long des cohabitations humaines.

\section{La diffusion des plantes et des savoirs médicinaux, un phénomène qui ne date pas d'hier}

18 Durant la période coloniale un très grand nombre d'espèces ont été disséminées sous les Tropiques, donnant lieu à des processus d'hybridation qui ont construit en grande partie les pharmacopées actuelles de ces régions (Benett \& Prance, 2000). Les populations indigènes présentes dans le bassin de l'Oyapock commerçaient avec les navires européens qui mouillaient dans la zone (Collomb \& van den Bel, 2014 ; Dupuy, 2012) et elles ont donc emprunté très tôt des espèces et des connaissances exogènes aux navigateurs. Deux exemples illustrent ces apports anciens : l'importance du manguier dans les pharmacopées locales ${ }^{14}$ alors qu'il s'agit d'une espèce originaire d'Inde et introduite à partir du 16 ème siècle en Amérique (Ferrao, 2015) et l'usage purgatif par les Créoles de la manne douce, exsudat d'un arbre d'Europe du sud (le frêne à manne, Fraxinus ornus ), aujourd'hui vendu en pharmacie, et que les marins européens du 18 ème siècle - qui l'utilisaient pour ses propriétés antirachitiques et pectorales - ont amené avec eux (Lalande, 1875 ; Luciani, 1926). 
19 Par la suite, au cours du 20 ème siècle, d'autres mouvements de population sont venus ajouter de la diversité botanique dans la région: par exemple, celui des orpailleurs créoles des premières ruées aurifères et des piroguiers saamaka ${ }^{15}$ qui les accompagnaient et dont descend toute une partie de la population créole actuelle (Laval, 2016 ; Mam-Lam-Fouck \& Anakesa, 2013; Pérez \& Archambeau, 2012) ${ }^{16}$. L'héritage de cette dernière migration s'exprime encore à travers l'utilisation de noms vernaculaires businenge pour désigner certaines plantes, à l'instar de singaafu (pour les espèces du genre Costus ), koo ati (Begonia glabra ) ou kuentu (Eryngium foetidum ).

Des phénomènes en cours d'interculturalisation ethnobotanique (Tareau, 2019) peuvent également être observés à Saint-Georges-de-l'Oyapock, impulsés en particulier par la diffusion d'espèces et d'usages associés en provenance du Brésil. En effet, cette enquête a permis de mettre en lumière le poids des plantes médicinales provenant $\mathrm{du}$ Brésil dans la pharmacopée oyapockoise : $10 \%$ des plantes médicinales consommées sont achetées ou collectées du côté brésilien. Les différentes communautés brésiliennes qui s'installent du côté français arrivent ainsi avec leurs propres connaissances tirées de la pharmacopée de leurs régions d'origine ; celles-ci finissent, au fur et à mesure des interactions quotidiennes entre habitants, par influencer les habitudes locales. Ces savoirs sont également partiellement relayés par les chaînes satellitaires brésiliennes captées à Saint-Georges-de-l'Oyapock. Certains remèdes populaires au Brésil le deviennent donc progressivement aussi du côté français, et font l'objet de flux transfrontaliers spécifiques, à l'instar de l'huile de copaïba ${ }^{17}$ (citée douze fois), qui est toujours achetée du côté brésilien, car elle n'est ni produite ni commercialisée en Guyane.

21 Les exemples de transmissions inter-communautaires sont potentiellement des facteurs de changement culturel à travers la diffusion d'espèces et de pratiques nouvelles dans les pharmacopées locales, contribuant à modifier progressivement les habitudes phyto-thérapeutiques et le paysage végétal local. De « nouvelles » pratiques, introduites et diffusées par les migrants, sont testées, modifiées au besoin et intégrées à la pharmacopée locale. Pour reprendre les termes consacrés par les études ethnobotaniques dans le domaine (Ladio et Albuquerque, 2014 ; Tareau, 2019), elles sont « relocalisées » dans un contexte nouveau et se "juxtaposent » à d'autres usages préexistants. Quelques témoignages recueillis sur le terrain peuvent être mentionnés afin d'illustrer le dynamisme sous-jacent de ce processus :

«J'étais malade, et c'est une dame créole qui m'a dit de prendre une plante appelée tiféy pour préparer un remède. Cette dame habite à l'entrée du quartier. » Femme d'origine palikur, 67 ans, quartier Petite Savane.

«C'est une collègue d'origine palikur qui m'a parlé de cette plante [Eupatorium ayapana]. Je la connaissais déjà, mais pour soigner d'autres choses. » Femme créole guyanaise, 23 ans, bourg.

C'est ainsi que plusieurs espèces recensées dans nos enquêtes (Plectranthus grandis (photo 2), Vernonia campestris, Morinda citrifolia) ne figurent pas dans l'ouvrage de référence Les pharmacopées traditionnelles de Guyane paru en 1986 et réédité en 2004 (Grenand et al., 2004), signe très probable de leur récente introduction dans la flore médicinale de Saint-Georges-de-l'Oyapock. Les personnes interrogées ont ainsi précisé que les deux premières avaient été amenées à Saint-Georges depuis le Brésil. 
Photographie 2 : Boldo, Plectranthus grandis, une plante introduite récemment depuis le Brésil. Village Onozo, 2017.

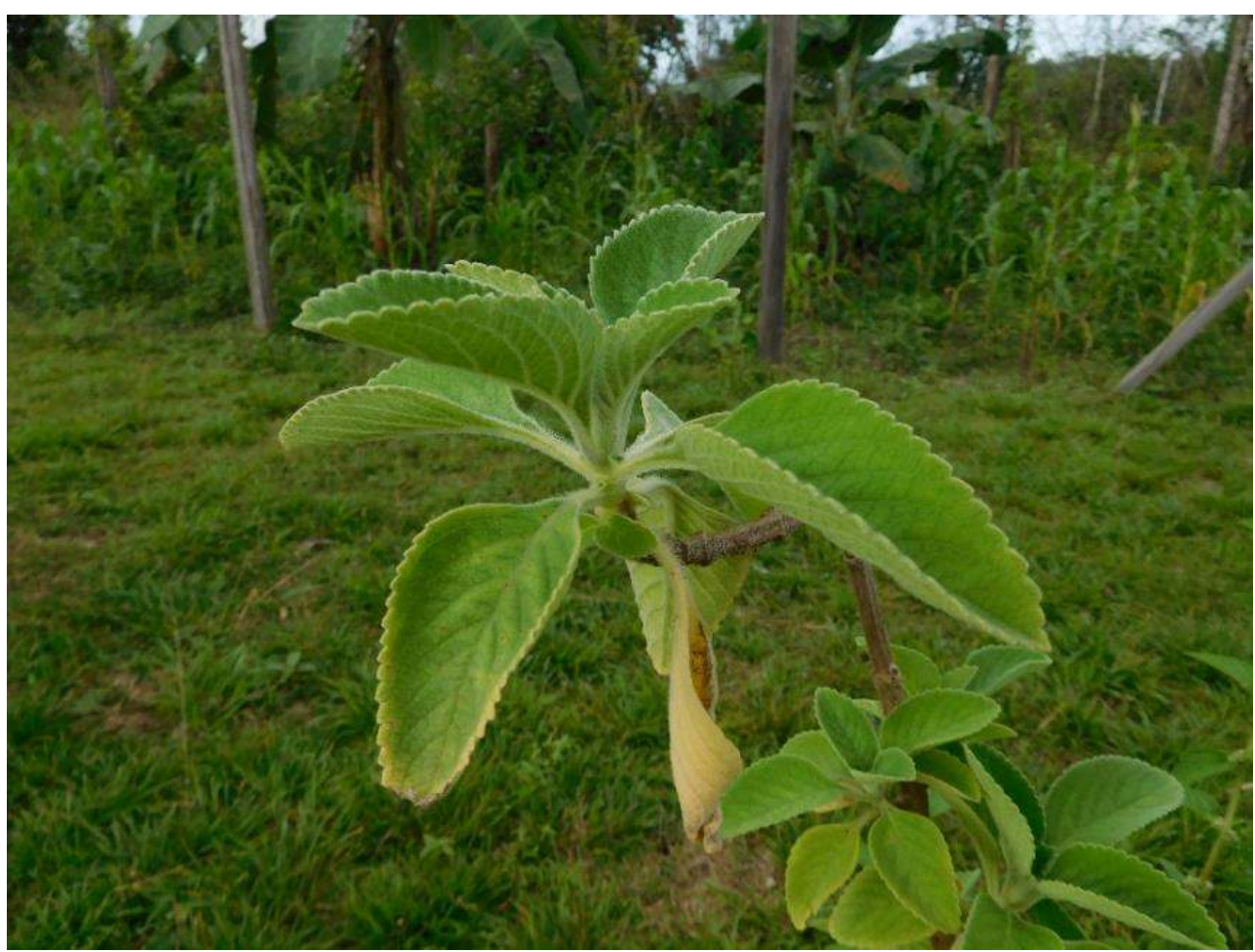

Des combinaisons entre remèdes locaux et biomédecine peuvent également apparaître : contre les fièvres fortes - notamment celles liées au paludisme - de l'acide acétylsalicylique (aspirine) est par exemple parfois ajouté à des décoctions de plantes connues pour leurs propriétés fébrifuges comme Quassia amara ${ }^{18}$ ou Phyllanthus amarus . Ces appropriations thérapeutiques mettent en exergue un pluralisme médical complexe, non seulement entre les différentes ethnomédecines, mais également entre celles-ci et la biomédecine. Enfin, des pratiques nouvelles peuvent aussi être inventées, contribuant à l'enrichissement des pharmacopées par l'ajout d'usages de novo (Odonne et al., 2011). Ainsi une femme palikur nous a-t-elle affirmé être la première personne de Saint-Georges à avoir fabriqué une huile médicinale avec des amandes de palmier dendé (palmier à huile africain, introduit comme ornemental dans la commune) :

«Je suis la première à avoir fait de l'huile avec des graines de palmier dendé (Elais guineensis). Désormais, beaucoup de personnes viennent m'en acheter : c'est très efficace contre les douleurs, tu peux te masser avec.» Femme d'origine palikur, 62 ans, quartier Petite-Savane.

24 Les processus de construction des pharmacopées sont donc complexes. Alors que les circulations des hommes entre et sur les continents ont favorisé et servent encore le déplacement des plantes, les échanges de savoirs suivent des routes moins visibles. À la faveur des cohabitations et ententes communautaires sur la zone frontalière et des besoins ponctuels en soins, les connaissances sur les usages des plantes se partagent, sont adoptées, testées, modifiées, contribuant au renouveau continuel des pharmacopées. 


\section{Les voyages transnationaux des hommes et des plantes : entre mobilités thérapeutiques et filières commerciales de plantes médicinales}

\section{Les circulations transfrontalières pour l'accès au soin}

Les trois communautés amérindiennes transfrontalières présentes à Saint-Georges-del'Oyapock (Galibi-marwono, Karipuna et Palikur) témoignent de déplacements réguliers et d'échanges réciproques avec leurs régions d'origine situées dans la région frontalière de l'Amapa, le long des fleuves Uaça, Kuripi et Urukaua. En cas de maladie grave par exemple, des pajé (chamanes) sont consultés dans les villages du Brésil ${ }^{19}$ ou se déplacent à Saint-Georges; ces déplacements épisodiques et bilatéraux revêtent une importance capitale dans les itinéraires de recours au soin et contribuent fortement à faire vivre les cultures indigènes de ces populations (Collomb, 2013).

"Quand on est gravement malade, on peut aller voir un pajé au village. Moi, je retourne à peu près deux fois chaque année à Manga, mon village de naissance. » Homme d'origine karipuna, 42 ans, quartier Bambou.

« Parfois, des gens viennent de Rokawa [dans l'État brésilien de l'Amapá] voir mes grands-parents. Ils nous racontent des histoires anciennes, celles de notre Peuple. »

Femme d'origine palikur, 23 ans, village Martin.

Par ailleurs, ces circulations médico-magiques sont parfois d'ordre interculturel puisque certains Créoles nous ont également confié s'être déplacés eux-mêmes dans les Terras Indigenas brésiliennes afin de consulter un pajé ${ }^{20}$ :

«On m'avait déclaré un cancer de la prostate. C'est une guérisseuse indienne de Kuripi [dans l'État de l'Amapá] qui m’a soigné avec des plantes. » Homme créole guyanais, 54 ans, piste Martin.

Ces mobilités dont l'objectif est d'obtenir des soins médico-magiques se font dans le sens inverse des déplacements de Brésiliens qui viennent au dispensaire de SaintGeorges pour se faire soigner. Ces mouvements transversaux contribuent à créer un espace circulatoire de santé sur la frontière.

Les Brésiliens résidant à Saint-Georges de l'Oyapock et originaires de villes plus au sud (Macapa, Belém et São Luís notamment) s'y rendent assez souvent afin de rendre visite à leurs proches ou faire des achats. Ils ramènent couramment dans leurs bagages des plantes médicinales achetées là-bas. Le grand marché de Ver-o-Peso à Belém, considéré comme le plus grand marché d'Amazonie, est notamment connu pour ses nombreuses échoppes de plantes médicinales (Soares Farias et Diniz Guerra, 2012 ; van den Berg, 1984).

\section{Les flux commerciaux de plantes transformées et de produits à base de plantes}

29 La présence d'une diaspora brésilienne en Guyane aussi bien que la diffusion progressive des pratiques de santé et consommations de plantes d'origine brésilienne ont engendré une demande accrue sur le territoire guyanais de phyto-médicaments brésiliens faisant aujourd'hui l'objet d'une circulation transamazonienne - voire transcontinentale - importante du Brésil vers la Guyane française. Impulsée et soutenue par l'État brésilien, une importante filière de transformation et de 
commercialisation de plantes médicinales existe au Brésil où, déjà en 2006, on comptabilisait 103 laboratoires produisant 367 médicaments phyto-thérapeutiques commercialisés dans l'ensemble du pays (Alves, 2013).

Des cartons de plantes séchées et conditionnées (entre autres: Artemisia vulgaris, Coutarea hexandra, Cuphea carthagenensis, Ginkgo biloba, Maytenus ilicifolia, Melissa officinalis, Peumus boldus, Rosmarinus officinalis, Ruta graveolens, Stryphnodendron adstringens ${ }^{21}$ ), provenant souvent d'États du sud du Brésil, situés à plusieurs milliers de kilomètres, parviennent ainsi à Oiapoque après un long trajet en camion à travers le pays, rendant notamment accessibles des espèces poussant en milieu tempéré à des populations vivant en Amazonie et induisant ainsi de fait un élargissement de la pharmacopée. C'est également le cas de grandes quantités d'huiles végétales ou d'oléorésines en provenance essentiellement des régions du Para, de l'Amazonas et du Maranhão où existe une importante filière extractiviste (Pinton \& Emperaire, 1992). Ces huiles ou résines transitent le plus souvent par les villes de Santarem, Belém puis Macapa avant d'être acheminées par la route jusqu'à Oiapoque dans des fûts ou des bidons et reconditionnées sur place (fig. 4). Les plantes séchées et produits transformés, qui bénéficient de circuits de distribution bien établis au Brésil, rejoignent donc la Guyane de manière informelle, par des filières relativement structurées. Ce type de circulations est quasiment unilatéral du Brésil vers la Guyane, la ville d'Oiapoque apparaissant comme un lieu de diffusion essentiel.

Figure 4 : Circulations des plantes et produits à base de plantes sur l'espace transfrontalier.

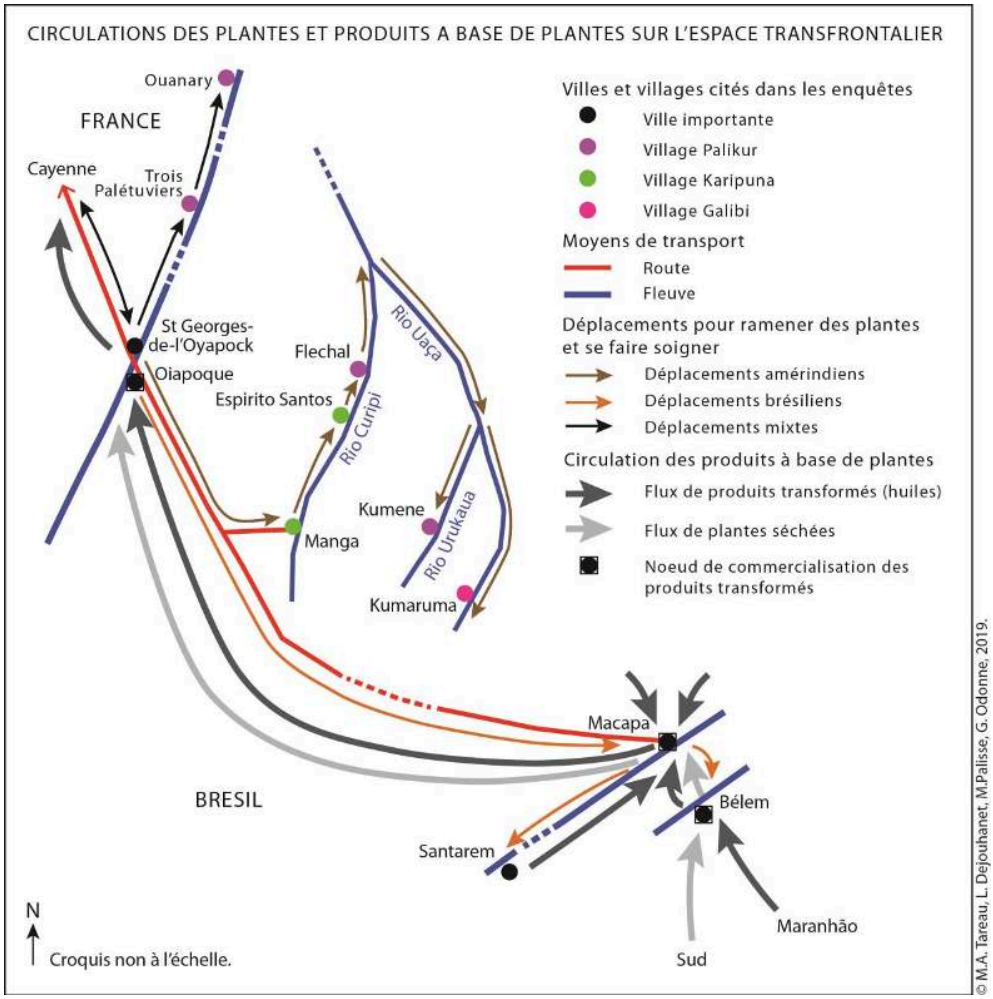

Revue francophone sur la santé et les territoires, Les circulations en santé : des produits, des savoirs, des personnes en mouvement 
Photos 3 et $4:$ Une des herboristeries d'Oiapoque vue extérieure et achalandage intérieur. Oiapoque, 2017.
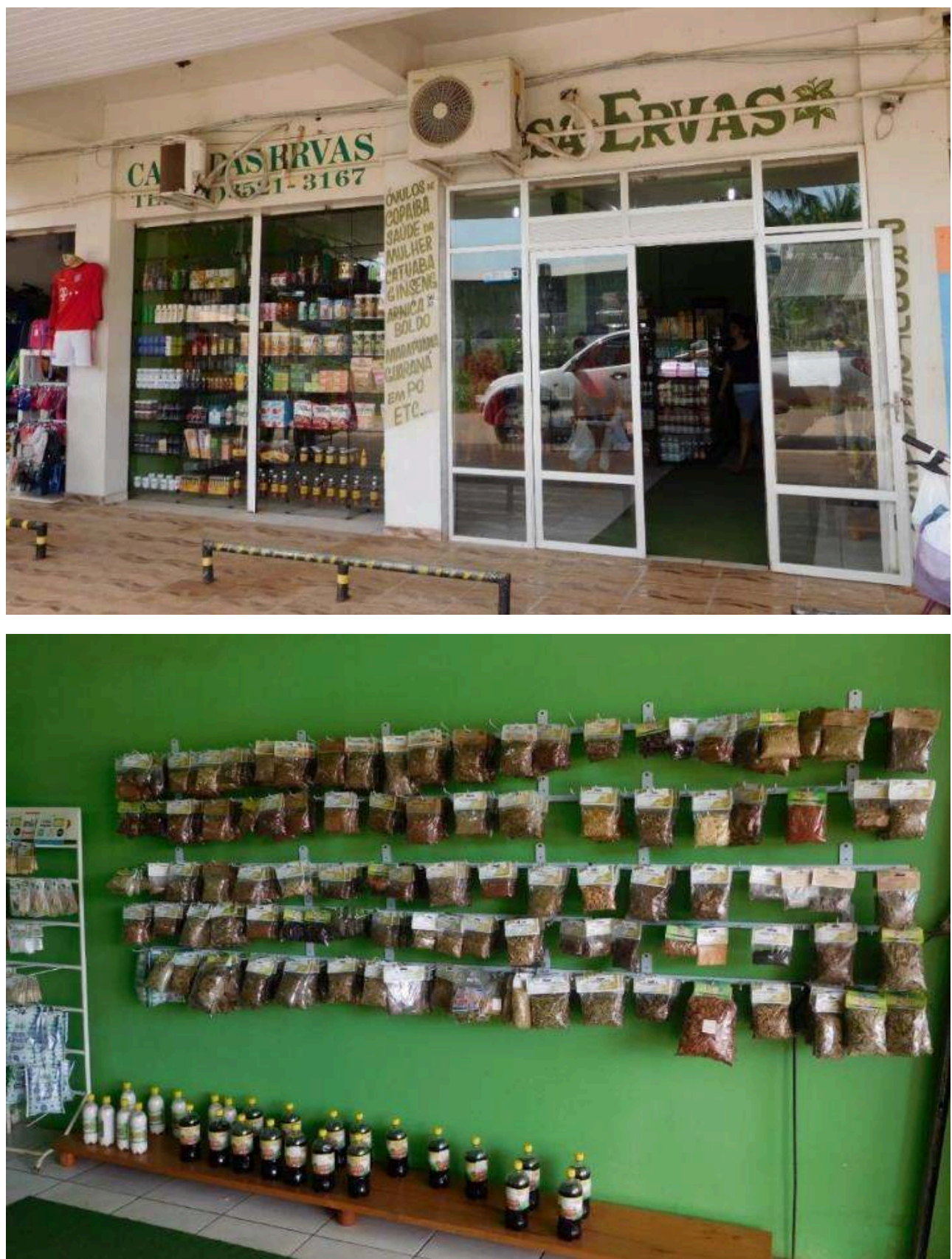
Photos 5 et 6 : Le marché d'Oiapoque, interface de vente pour les plantes et huiles végétales. Oiapoque, 2017.
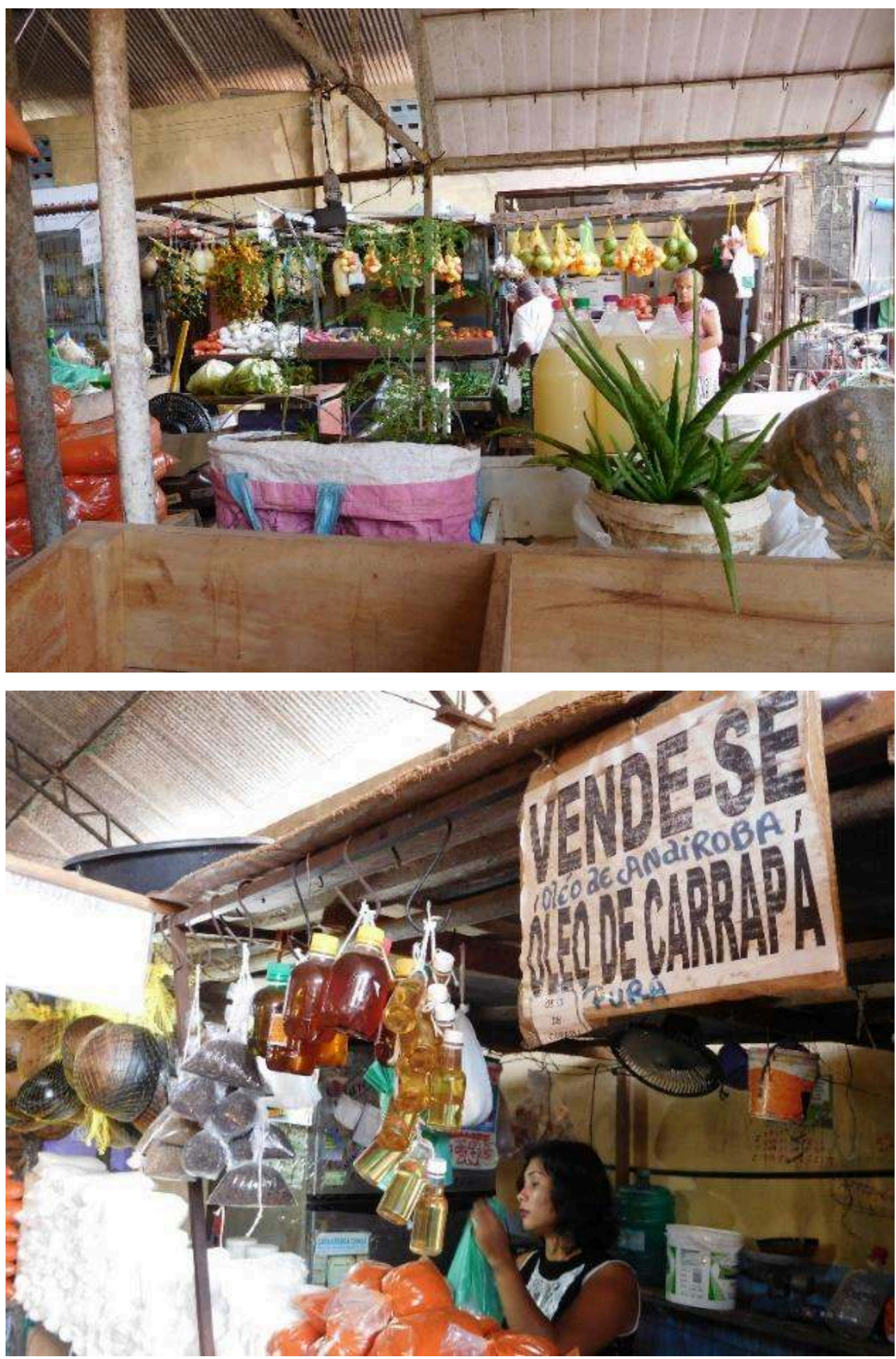

Oiapoque, interface de diffusion des plantes médicinales vers le marché guyanais

31 Ces flux de plantes médicinales et de phyto-produits en provenance d'autres régions du Brésil aboutissent à Oiapoque où ils sont ensuite commercialisés et diffusés vers la 
Guyane, dans un cadre essentiellement domestique - et donc informel. Cette ville frontalière, à la fois carrefour et marge, véritable nœud de connexions multimodales, constitue en effet un lieu important d'approvisionnement en tous types de produits : agroalimentaires, manufacturés, médicinaux... pour les habitants de Saint-Georges-del'Oyapock et même de l'ensemble du littoral guyanais. On vient de Cayenne jusqu'à Oiapoque faire des courses bon marché, de produits de toute sorte ou typiquement brésiliens (charcuterie brésilienne, produits de beauté, accessoires de mode, noix du Brésil...), en empruntant une pirogue à Saint-Georges ou en traversant le pont avec son véhicule.

32 Les deux "interfaces" principales de vente des plantes à Oiapoque sont les herboristeries et le marché central. Plusieurs herboristeries (photos 4), bien approvisionnées, proposent ainsi des plantes séchées et conditionnées et divers produits phyto-médicinaux. Le métier d'herboriste étant aujourd'hui interdit en France ${ }^{22}$, à la différence du Brésil, de nombreux consommateurs guyanais se rendent à Oiapoque afin de bénéficier des conseils délivrés par ces officines et acheter des remèdes introuvables du côté français, tels que le sirop tonique de guarana ( Paullinia cupana) ou le "lait d'amapa", latex d'une espèce forestière amazonienne (probablement Parahancornia fasciculata) consommée en cure fortifiante.

33 Le marché quant à lui propose plus particulièrement des plantes fraîches ( barbosa , Aloe vera ; arruda, Ruta graveolens ; mastruz, Chenopodium ambrosioides ; chicorea, Eryngium foetidum ) et des huiles végétales (carapa, coco, copaïba) (photos 5). Si les herboristeries attirent les consommateurs français en quête d'huiles et de plantes séchées certifiées, le marché, lui, contribue à la diffusion de produits plus artisanaux et de végétaux vers la Guyane.

\section{Conclusion}

En jouant sur les échelles d'analyse, cette étude montre à quel point les circulations de plantes et de savoirs, qu'elles soient transnationales ou micro-locales (à l'échelle du quartier), composent une réalité forte de l'espace culturel et social de Saint-Georgesde-l'Oyapock. Les modes de circulations des savoirs, des plantes et des personnes ainsi mis en évidence s'inscrivent en effet dans les caractéristiques de fonctionnement d'un système spatial transfrontalier qui agit ici selon le modèle du «métamorphisme de contact » tel que décrit par J.P. Renard et P. Picouet (1993, cité in Beucher \& Reghezza, 2017). Celui-ci repose à la fois sur des mobilités transfrontalières, une zone de bilinguisme et des relations amicales et familiales qui permettent la constitution d'un espace culturel et vécu. A ceci s'ajoutent une diffusion transnationale d'informations écrites et radio-télévisées et des flux de consommateurs qui traversent la frontière pour s'approvisionner dans le pôle urbain voisin.

La rive brésilienne de la frontière entre le Brésil et la Guyane française peut être qualifiée de "tinctoriale» tant il apparaît qu'elle détient une influence forte et quasiment à sens unique, sur les pharmacopées de Saint-Georges-de-l'Oyapock, tant en termes de provenance des plantes médicinales utilisées localement (plus de $10 \%$ ) que d'introduction d'espèces et d'usages nouveaux venant s'hybrider avec les phytothérapies locales et parfois s'implanter durablement. Ces circulations font écho aux échanges de semences, boutures et tubercules déjà étudiés dans la région (Grenand, 2011 ; Martins Faure, 2010), qui viennent eux aussi enrichir le patrimoine bioculturel et 
végétal des deux rives. Le poids du Brésil dans ces échanges est sans doute à lier à l'immensité du territoire brésilien qui permet l'accès à une multitude de plantes médicinales ou alimentaires et à la politique volontariste de ses gouvernements en matière de développement de phyto-produits. Elle est aussi à rattacher à l'histoire particulière de cette frontière franco-brésilienne qui a jusqu'à aujourd'hui fonctionné comme une interface entre les cultures.

Cette recherche montre ainsi que les frontières en tant que zones d'échanges et d'influences mutuelles sont des zones de diffusion importantes des phytothérapies et des ethnomédecines. Elle compose une partie d'un travail plus large sur les circulations bioculturelles intercommunautaires à l'échelle de la Guyane française et de ses voisins.

\section{BIBLIOGRAPHIE}

Alexiades, M.N., 2009. Mobility and Migration in Indigenous Amazonia: Contemporary Ethnoecological Perspectives. Berghahn Books.

Alves, L.F., 2013. Produção de Fitoterápicos no Brasil: História, Problemas e Perspectivas. Rev. Virtual Quím. 5, 450-513.

Audebert, C., 2017. La diaspora haïtienne : Territoires migratoires et réseaux. Presses universitaires de Rennes.

Balick, M.J., Kronenberg, F., Ososki, A.L., Reiff, M., Fugh-Berman, A., Bonnie, O., Roble, M., Lohr, P., Atha, D., 2000. Medicinal plants used by latino healers for women's health conditions in New York City. Econ. Bot.

Benett, B., Prance, G., 2000. Introduced plants in the indigenous pharmacopeias of the northern South America. Econ. Bot. 1, 90-102.

Benoist, J., 1996. Soigner au pluriel : essais sur le pluralisme médical. Karthala.

Bertani, S. et al., 2012. New findings on Simalikalactone D, an antimalarial compound from Quassia amara L. (Simaroubaceae). Experimental Parasitology 130(4): 341-47.

Beucher, S., Reghezza, M., 2017, La géographie : pourquoi ? Comment ? Hatier.

Blancodini, P., Tabarly, S., 2010. Les frontiers externes et les limites internes en Guyane, entre fragmentation, ruptures et interfaces. Géoconfluences.

Bochaton, A., 2018. Intertwined Therapeutic Mobilities : Knowledge, plants, healers on the move between Laos and the U.S.. Mobilities, Taylor \& Francis (Routledge), 1-17.

Bochaton, A., Lefebvre, B., 2010. Interviewing elites. Perspectives from medical tourism sector in India and in Thailand. In Hall, M. (éd.) Fieldwork in Tourism. Methods, Issues and Reflections, Routledge New York, 85-95.

Bochaton, A., 2009. « Recours aux soins transfrontaliers et réseaux informels : l'exemple laothailandais ». 
Bochaton, A., Lefebvre, B., 2008. The rebirth of the hospital: Heterotopia and medical tourism in Asia. In Winter, T., Teo, P., Chang, T.C. (eds) Asia on Tour. Exploring the rise of Asian tourism , Routledge New York, 97-108

Boudoux d'Hautefeuille, M., 2008. Entre marge et interface : recompositions territoriales à la frontière franco-brésilienne (Guyane/Amapa), Thèse de doctorat en géographie, Université des Antilles et de la Guyane.

Bruckmann, L., 2017. « Les territoires du fleuve » : une analyse par l'image de l'intégration territoriale du fleuve Sénégal. EchoGéo.

Bruneau, M., 2010. Diasporas, transnational spaces and communities. Diaspora Transnationalism Concepts Theor. Methods 35-50.

Candelise, L., 2013. Patrimonialisation des savoirs médicaux : vers une reconfiguration des ressources thérapeutiques. Introduction au dossier thématique. Anthropol. Santé Rev. Int. Francoph. Anthropol. Santé.

Carreño Calderón, A., 2013. Revisiter les frontières et réparer l'histoire. Itinéraires thérapeutiques et processus de sauvegarde de la médecine traditionnelle andine dans un contexte transnational. Anthropol. Santé Rev. Int. Francoph. Anthropol. Santé.

Ceuterick, M., Vandebroek, I., Pieroni, A., 2011. Resilience of Andean urban ethnobotanies: A comparison of medicinal plant use among Bolivian and Peruvian migrants in the United Kingdom and in their countries of origin. J. Ethnopharmacol. 136, 27-54.

Ceuterick, M., Vandebroek, I., Torry, B., Pieroni, A., 2008. Cross-cultural adaptation in urban ethnobotany: The Colombian folk pharmacopoeia in London. J. Ethnopharmacol. 120, 342-359.

Collomb, G., 2013. «Indiens » ou « Brésiliens » ? Mobilités karipuna vers Cayenne (Guyane française). Rev. Eur. Migr. Int. 29, 113-131.

Collomb, G., van den Bel, M., 2014. Entre 2 mondes, Amérindiens \& Européens : sur les côtes de Guyane, avant la colonie, 1560-1627. CTHS.

Craig., S.R., 2013. Les différents visages d'un guérisseur himalayen. in : L. Pordié, E. Simon (eds) Les nouveaux guérisseurs. Biographies de thérapeutes au temps de la globalisation. p 155-180, EHESS éditions.

Crété, C., 2015. Piroguiers, flux et structuration de l'espace : les piroguiers de l'Oyapock et le pont binational (Guyane, France et Etat de l'Amapá, Brésil). Mémoire de Master 2, Université Joseph Fournier Grenoble.

de Oliveira, T.C.M., 2009. Frontières en Amérique latine : réflexions méthodologiques. Espac. Sociétés 19-33.

Dejouhanet, L., 2014. Supply of Medicinal Raw Materials: The Achilles' Heel of Today's Manufacturing Sector for Ayurvedic Drugs in Kerala. Asian Medicine. Tradition and Modernity, Vol. 7, 3.

Dejouhanet, L., 2009. L'Ayurveda. Mondialisation d'une médecine traditionnelle indienne. EchoGéo.

Dupuy, F., 2012. Les arpenteurs des confins : explorateurs de l'intérieur de la Guyane, 1720-1860. CTHS.

Ferrao, J. et M., 2015. Le Voyage des plantes et les grandes découvertes. Chandeigne. 
Filippi, M., 2016. Réglementations et pratiques fluviales sur le bassin du bas Oyapock, un territoire aux confins de l'Amazonie.

Fleury, M., 1997. À propos de I'intérêt médicinal du baume de Copahu. Acta Bot. Gallica 144, 473479.

Grenand, F., 2011. Un pont entre la France et le Brésil : l'Observatoire Hommes/Milieux sur le fleuve Oyapock, Rayonnement du CNRS n56 juin 2011.

Grenand, F., 2012. Enjeux de territoires sur une frontière méconnue, Confins. Revue francobrésilienne de géographie / Revista franco-brasilera de geografia, 16.

Grenand, P., Moretti, C., Jacquemain, H., Prévost, M.-F., 2004. Pharmacopées traditionnelles en Guyane : créoles, wayãpi, palikur. IRD Editions.

Houël, E. et al., 2009. Quassinoid constituents of Quassia amara L. leaf herbal tea. Impact on its antimalarial activity and cytotoxicity. Journal of Ethnopharmacology 126(1): 114-18.

Hoyez, A.-C., 2011. L'espace-monde du yoga : De la santé aux paysages thérapeutiques mondialisés. Presses universitaires de Rennes.

Kujawska, M., Hilgert, N.I., 2014. Phytotherapy of Polish migrants in Misiones, Argentina : Legacy and acquired plant species. J. Ethnopharmacol. 153, 810-830.

Ladio, A.H., Albuquerque, U.P., 2014. The concept of hybridization and its contribution to urban ethnobiology. Ethnobiol. Conserv. 1-9.

Lalande, E., 1875. Essai sur les mannes pharmaceutiques et les frênes qui les produisent : Thèse présentée à l'Ecole supérieure de pharmacie de Montpellier, le 9 juin 1875 (PhD Thesis). Imprimerie centrale du Midi, Ricateau, Hamelin et Cie.

Laval, P., 2016. Captures estuariennes : une ethnoécologie de la pêche sur le bas Oyapock (frontière franco-brésilienne). Thèse de doctorat, Museum national d'histoire naturelle - MNHN Paris.

Leonti, M., 2011. The future is written: Impact of scripts on the cognition, selection, knowledge and transmission of medicinal plant use and its implications for ethnobotany and ethnopharmacology. J. Ethnopharmacol. 134, 542-555.

Leslie, C., 1989. Indigenous pharmaceuticals, the capitalist world system, and civilization. Kroeber Anthropol. Soc. Pap. 69, 23-31.

Leslie, C., 1980. Medical pluralism in world perspective [1]. Soc. Sci. Med. [B] 14, 191-195.

Leslie, C., Leslie, C.M., Young, A., 1992. Paths to Asian medical knowledge. Univ of California Press.

Letniowska-Swiat, S., 2012, Oyapock, un pont trop loin ? Un pont pour quoi ? ", dossier « La frontière, discontinuités et dynamiques, Géoconfluences.

Lieutaghi, P. 1991. Plante compagne. Editions du Musée d'Histoire de Neuchâtel.

Lorenzi, H., Matos, F.J., 2002. Plantas medicinais no Brasil: nativas e exóticas. Instituto Plantarum de Estudos da Flora, Brésil.

Luciani, D. 1926. Le frêne à Manne, la manne. Office national des matières premières végétales pour la droguerie, la pharmacie, la distillerie et la parfumerie.

Mam-Lam-Fouck, S., Anakesa, A., 2013. Nouvelle histoire de la Guyane, Ibis Rouge Editions. 
Martins Faure, L., 2010. Les dynamiques spatiales en zone frontalière : contributions vers un diagnostic de l'économie des petits exploitants agricoles, Rapport pour le compte de l'OHM Oyapock.

Mercan, A., 2012. La route du cordyceps. Autrepart.

Moullé F. (dir.), 2017. Frontières. Presses Universitaires de Bordeaux.

Mung, E.M., 1992. Dispositif économique et ressources spatiales : éléments d'une économie de diaspora. Rev. Eur. Migr. Int. 8, 175-193.

Nicolas, T., 2016. «Frontières, migrations et reconfigurations territoriales en Guyane », in Collomb Gérard et Mam Lam Fouck Serge (direction), Mobilités, ethnicités, diversité culturelle : la Guyane entre Surinam et Brésil. Eléments de compréhension de la situation guyanaise, Matoury, Ibis Rouge Editions, 273-298.

Nouailhat, R., 2010. L'Oyapock, frontière impossible. Médium, 118-123.

Odonne, G., 2014. Ethnobotany of Amazonia. In Encyclopaedia of the History of Science, Technology and Medicine in the Non-Western Cultures.

Odonne, G., Berger, F., Stien, D., Grenand, P., Bourdy, G., 2011. Treatment of leishmaniasis in the Oyapock basin (French Guiana): A K.A.P. survey and analysis of the evolution of phytotherapy knowledge amongst Wayãpi Indians. J. Ethnopharmacol. 137, 1228-1239.

Ososki, A.L., Balick, M.J., Daly, D.C., 2007. Medicinal plants and cultural variation across Dominican rural, urban, and transnational landscapes. Travel. Cult. Plants Ethnobiol. Ethnopharmacy Migr. 7, 14-38.

Pérez, P., Archambeau, O., 2012. Architectures et paysages de Saint-Georges de l'Oyapock. OHM Oyapock, CNRS Guyane (Cayenne).

Phillips, O., Gentry, A.H. 1993. « The useful plants of Tambopata, Peru: I. Statistical hypotheses tests with a new quantitative technique ». Economic Botany 47(1), 15-32.

Pieroni, A., Quave, C.L., Giusti, M.E., Papp, N., 2012. “We are Italians!”: the hybrid ethnobotany of a Venetian diaspora in Eastern Romania. Hum. Ecol. 40, 435-451.

Pieroni, A., Sheikh, Q.-Z., Ali, W., Torry, B., 2008. Traditional medicines used by Pakistani migrants from Mirpur living in Bradford, Northern England. Complement. Ther. Med. 16, 81-86.

Pinton, F., Emperaire, L., 1992. L'extractivisme en Amazonie brésilienne : un système en crise d'identité. Cah. Sci. Hum. 28, 685-703.

Police, G., 2010. Eudorado. Le discours brésilien sur la Guyane française. Ibis Rouge Editions. Pordié, L., 2013. Les pérégrinations de Dolma Tsering. Itinéraire transnational d'un médecin tibétain. Nouv. Guérisseurs Biogr. Thérapeutes Au Temps Glob. Paris EHESS, 155-180.

Pordié, L., 2002. La pharmacopée comme expression de société. Une étude himalayenne. Sources Savoir Aux Médicam. Futur, 183-94.

Pordié, L., Simon, E., 2013. Les nouveaux guérisseurs. Biographies de thérapeutes au temps de la globalisation. EHESS.

Price, R., 2013. Les Premiers Temps : la conception de l'histoire des Marrons Saamaka. Vents d'ailleurs.

Reiff, M., O'Connor, B., Kronenberg, F., Balick, M., Lohr, P., Roble, M., Fugh-Berman, A., Johnson, K.D., 2003. Ethnomedicine in the Urban Environment: Dominican Healers in New York City. Hum. Organ. $62,12-26$. 
Ruffray, S. de, 2000. De la marginalité territoriale à la recomposition territoriale « marginale ». Rev. Géographique Est 40.

Sakoyan, J., 2012. Les mobilités thérapeutiques. Bilan et perspectives depuis les Comores. Anthropol. Santé Rev. Int. Francoph. Anthropol. Santé.

Saxer, M., 2009. Herbs and Traders in Transit: Border Regimes and the Contemporary TransHimalayan Trade in Tibetan Medicinal Plants. Asian Medicine. Tradition and Modernity, Vol. 5, 317-339.

Soares Farias, E., Diniz Guerra, G.A., 2012. Plantas medicinais, ervas aromaticas e produtos para usos imateriais na Féria do 25, em Belem do para. Terceira Margem Amazon. 1, 207-218.

Tareau, M.A., 2019. Les pharmacopées métissées de Guyane : ethnobotanique d'une phytothérapie en mouvement. Thèse de doctorat, Université de Guyane.

Tareau, M.A., Dejouhanet L., Odonne G., Palisse M., Ansoe C., 2019. Penser la cueillette de plantes médicinales sauvages dans des sociétés en transition : le cas guyanais. EchoGéo 47.

Tareau, M.A., Palisse, M., Odonne, G., 2017. As vivid as a weed.... Medicinal and cosmetic plant uses amongst the urban youth in French Guiana. J. Ethnopharmacol. 203, 200-213.

Thebaux, P., 2015. Le pont de l'Oyapock : Quels discours et quelles représentations des médias et des riverains? Mémoire de Master 2, Université de Montpellier 2.

Théry, H., 2015. À quoi sert la Guyane ? Outre-Terre 43, 211.

Tritsch, I., Gond, V., Oszwald, J., Davy, D., Grenand, P., 2012. Dynamiques territoriales des Amérindiens wayãpi et teko du moyen Oyapock, Camopi, Guyane française. Bois For. Trop. 49- 61. van Andel, T., Westers, P., 2010. Why Surinamese migrants in the Netherlands continue to use medicinal herbs from their home country. J. Ethnopharmacol. 127, 694-701.

van den Berg, M.E., 1984. Ver-o-Peso : the ethnobotany of an amazonian market., in : Ethnobotany in the Neotropics. G. T. Prance \& J. A. Kallunki, 140-149.

Vandebroek, I., Balick, M.J., 2012. Globalization and Loss of Plant Knowledge : Challenging the Paradigm. PLoS ONE 7, e37643.

Vilayleck, E., 2002. Ethnobotanique et médecine traditionnelle créoles : contribution à l'étude du lexique de l'habitation. Ibis rouge Ed., Presses universitaires créoles GEREC/F.

Voeks, R.A., 2007. Are women reservoirs of knowledge? Gender, ethnobotany and globalization in northeast Brazil. Singap. J. Trop. Geogr. 28, 7.

Volpato, G., Godinez, D., Beyra, A., 2008. Migration and ethnobotanical practices : the case of tifey among haitians immigrants in Cuba. Hum. Ecol., 43-53.

\section{NOTES}

1. Les Amérindiens galibi-marwono et karipuna du bas Oyapock parlent le khéuol, une langue très proche du créole guyanais.

2. On estime à approximativement 600 usagers par jour le trafic fluvial entre SaintGeorges et Oiapoque (Crété, 2015).

3. La continuité du milieu naturel est caractérisée par un territoire transfrontalier majoritairement recouvert d'une forêt équatoriale humide et dense. 
4. La route entre Oiapoque et Macapa date des années 1970 et celle entre Cayenne et Saint-Georges-de-l'Oyapock n'a été ouverte qu'en 2003. Avant cela, les liaisons ne se faisaient que par voie maritime ou aérienne, onéreuses et peu nombreuses.

5. Comme pour les autres ethnosciences, le terme "ethnobotanique " admet une double acception, désignant tantôt le domaine d'étude, tantôt l'objet d'étude. En effet, il s'agit soit de «l'ensemble des savoirs et des pratiques d'un groupe humain sur son environnement végétal» (Vilayleck, 2002) soit, pour reprendre la définition très précise donnée par l'ethnobotaniste français Lieutaghi de «l'attention portée à tous les aspects des rapports anciens et actuels des sociétés avec la plante comme élément du territoire, comme nom, comme aliment, remède, matériau des techniques, signe, symbole, vecteur de pouvoirs, support de croyances, etc » (Lieutaghi, 1991).

6. La complexité législative autour des recherches en ethnobotanique au Brésil nous a encouragés à nous concentrer sur la rive française.

7. Cette loi ne concerne que les connaissances traditionnelles propres aux peuples amérindiens et bushinenge.

8. Au début 2019, elle n'était toujours pas opérationnelle en ce qui concerne les savoirs traditionnels liés à la biodiversité.

9. http://www.ethnobiology.net/what-we-do/core-programs/ise-ethics-program/ code-of-ethics/

10. Leur nombre est estimé à 20000 personnes par le consulat du Brésil (Police, 2010).

11. Les échantillons botaniques prélevés sur le terrain ont été conditionnés, séchés, conservés et identifiés à l'Herbier IRD de Cayenne.

12. Cette importance des femmes a déjà été montrée dans d'autres études dans le nord de l'Amérique du sud (Tareau et al., 2017 ; Voeks, 2007).

13. Les connaissances dont la provenance est mixte n'ont pas été prises en compte dans ce calcul, laissant imaginer une part encore plus importante de la transmission féminine...

14. L'écorce de manguier est notamment utilisée dans la médecine populaire palikur en décoction anti-diarrhéique. Des prélèvements d'écorce peuvent ainsi fréquemment être observés localement sur les troncs de manguiers.

15. Il s'agit d'un groupe ethno-culturel businenge, ou Noir-Marron, c'est-à-dire descendant d'esclaves qui avaient fui les plantations surinamaises au cours des 17 ème et 18 ème siècles pour s'installer le long du Maroni.

16. On estime à environ 300 personnes le nombre de piroguiers marrons surinamais qui se sont installés sur l'Oyapock dans la première moitié du 20 ème siècle afin de ravitailler les mines d'or de l'époque (Laval 2016 ; Price, 2013), donnant naissance à un groupe culturel distinct (fortement métissé aujourd'hui avec les populations créoles et amérindiennes) dont le culte des ancêtres, la médecine traditionnelle, l'architecture et certaines pratiques linguistiques ont subsisté (Pérez and Archambeau, 2012).

17. Il s'agit d'une oléorésine constituant un cicatrisant très réputé dans toute l'Amazonie (Odonne, 2014) et à la base d'un baume populaire en Europe au 19 ème siècle (Fleury, 1997).

18. Espèce la plus couramment citée à Saint-Georges-de-l'Oyapock, Quassia amara a fait l'objet de plusieurs études pharmacologiques ayant souligné son activité antipaludéenne (Bertani et al., 2012 ; Houël et al., 2009). 
19. Ces villages sont intégrés dans des réserves ( Terras Indigenas ) qui veillent à la préservation des valeurs culturelles autochtones.

20. Terme d'origine Tupi-guarani caractérisant dans l'est de l'Amazonie le thérapeute le plus proche du concept de chamane.

21. Ces espèces ont été vues sous forme de plantes séchées et conditionnées en sachets dans une herboristerie d'Oiapoque. Les taxons présumés proviennent de l'ouvrage de Lorenzi et Matos (2002), par correspondance aux noms vernaculaires inscrits sur les paquets.

22. Les plantes médicinales relèvent, en France, du monopole pharmaceutique, c'est-àdire qu'elles ne peuvent être dispensées au public qu'en pharmacie, conformément à l'article L. $4211-1 / 5^{\circ}$ du Code de la santé publique, à l'exception toutefois d'un certain nombre de plantes « libérées » de ce monopole.

\section{RÉSUMÉS}

Les pharmacopées sont, à l'image de toute production culturelle, des objets éminemment vivants et dynamiques qui se transforment et se réinventent constamment, au fil des contacts et échanges incessants qui se jouent entre les différents groupes humains. Les plantes médicinales sont, elles aussi, des «objets bioculturels» (Pordié, 2002) par la richesse et la complexité des relations que les sociétés entretiennent avec elles, elles aussi toujours en évolution.

En Guyane, et en particulier sur les espaces transfrontaliers, les interactions culturelles sont constantes. Les plantes et leurs usages circulent entre les communautés, participant au renouvellement et à l'hybridation continuelle des phytothérapies. Ces flux matériels et immatériels composent la réalité culturelle des espaces transfrontaliers du plateau des Guyanes. Cet article se focalise sur la frontière franco-brésilienne matérialisée par le fleuve Oyapock. À la lumière d'entretiens menés dans la ville de Saint-Georges-de-l'Oyapock, nous proposons une description exploratoire des flux ethnobotaniques en contexte transfrontalier, en nous intéressant aux plantes médicinales échangées, à la diffusion des savoirs associés et donc aux modes de circulation des plantes et des connaissances. Cette étude compose une partie d'une recherche plus large sur les circulations bioculturelles à l'échelle de la Guyane et de ses voisins.

Pharmacopoeias are, like any kind of cultural production, living and dynamic objects that constantly change and reinvent themselves through continuous contacts and exchanges between different social groups. Medicinal plants are "biocultural objects" (Pordié, 2002) because of the richness and the complexity of relationships that societies develop with them. In French Guiana, especially in its cross-border areas, cultural interactions are common. Plants and associated knowledge circulate among communities, contributing to a permanent renewal and a continuous hybridization of herbal medicines. These material and immaterial flows form the cultural reality of cross-border areas on the Guiana Shield.

This paper focuses on the border between French Guiana and Brazil, which is made tangible by the Oyapock River. This border is a relatively recent political construction as it was only established in 1900 after a Swiss arbitration ended a long diplomatic conflict between France and Brazil. Even if a process of "rebordering" (Moullé, 2017) has been observed lately - despite the opening of the Oyapock bridge - the two sides of the River are part of the same dynamic cultural 
space since both share a relative remoteness from their respective centre. As well, constant social, economic and cultural circulations take place between the French town of Saint-Georgesde-l'Oyapock and the Brazilian town of Oiapoque. In this context, ethnobotanical circulations namely, exchanges of medicinal plants and associated knowledge - are a fair illustration of the shared cultural dimension between people.

Based on interviews with 23 inhabitants of the French town of Saint-Georges-de-l'Oyapock, the paper offers an exploratory description of ethnobotanical flows in the context of the crossborder area. It lists the medicinal plants used in Saint-Georges and analyses the diffusion of associated knowledge between the different communities. Results show that $92 \%$ of the 139 identified plants are used by several communities, and $40 \%$ of them are used among four to five different social groups. While most of the used plants are exotic species, their use is quite transversal to the socio-cultural components of Saint-Georges population. Nevertheless, the analysis shows that most of the knowledge transmission about medicinal plants happens inside the same community and via inter-generational means. The use of the same species and medicinal knowledge by different groups is therefore the result of a long-term process. Currently, it seems that early-established communities are more likely to share their knowledge with other groups than newly arrived groups, who integrate local uses to their own pharmacopoeias but seldom share their knowledge. Even though inter-community exchanges are discreet, they still contribute to vivid dynamics of local pharmacopoeias, illustrated by current ethnobotanical "interculturalisation" (Tareau, 2019), relocation and juxtapositions of phytotherapeutical practices, as well as new practices based on combinations between treatments from different origins.

The cross-border area is therefore a place of cultural hybridization, in a context of intensive circulations of people and goods between two socio-political systems (Map 1). Practices of medical pluralism explain some of the to-and-fro moves of people on the border: while Brazilians come to Saint-Georges' dispensary for health treatment, French Amerindians and Creoles visit Terras Indigenas villages in Brazil for consulting pajés. The pajés may also come to Saint-Georges to visit their family and treat sick persons. At the regional scale, circulations for access to care contribute to the delineation of a large health circulation area on the border. The latter also acts as an attraction factor for flows of processed medicinal plants, which find in Oiapoque an interface for entering the Guianese health market (see Map 1). Several factors explain this regional export capacity of Brazilian herbal goods: the presence of Brazilian diaspora and the diffusion of its pharmacopoeia in French Guiana along with the marketing strength of Brazilian herbal pharmacies and the presence of herbalists in Oaipoque, although they are not allowed in France. All these contribute to the development of flows of dried medicinal plants and processed herbal items from inner and Southern Brazil to French Guiana, and therefore to the relentless evolution of local pharmacopoeias.

INDEX

Mots-clés : circulation, ethnobotanique, plantes médicinales, frontière

Financement http://dx.doi.org/10.13039/501100001665

Keywords : circulation, ethnobotany, French Guiana, Brazil, medicinal plants, boundarie Index géographique : Brésil, Guyane française 


\section{AUTEURS}

\section{MARC-ALEXANDRE TAREAU}

Doctorant en ethnobotanique, Université de Guyane, USR mixte LEEISA

\section{LUCIE DEJOUHANET}

Maître de conférences en géographie, Université des Antilles, AIHP-GEODE EA 929, Institut Convergences Migrations

\section{MARIANNE PALISSE}

Maître de conférences en anthropologie, Université de Guyane, USR mixte LEEISA

\section{GUILLAUME ODONNE}

Chargé de recherche CNRS en ethnobiologie, USR mixte LEEISA 